\title{
The DSM-5 Levels of Personality Functioning and Severity of Iranian Patients With Antisocial and Borderline Personality Disorders
}

\author{
Mehdi Amini ${ }^{1}$; Abbas Pourshahbaz ${ }^{2, *}$; Parvaneh Mohammadkhani ${ }^{2}$; Mohammad Reza \\ Khodaie Ardakani ${ }^{3}$; Mozhgan Lotfi ${ }^{4}$ \\ ${ }_{1}^{1}$ Behavioral Sciences Research Center, Baqiyatallah University of Medical Sciences, Tehran, IR Iran \\ ${ }^{2}$ Department of Clinical Psychology, University of Social Welfare and Rehabilitation Sciences, Tehran, IR Iran \\ ${ }^{3}$ Department of Psychiatry, University of Social Welfare and Rehabilitation Sciences, Tehran, IR Iran \\ ${ }^{4}$ Mental Health Research Center, Tehran Institute of Psychiatry, School of Behavioral Sciences and Mental Health, Iran University of Medical Sciences, Tehran, IR Iran \\ ${ }^{*}$ Corresponding Author: Abbas Pourshahbaz, Department of Clinical Psychology, University of Social Welfare and Rehabilitation Sciences, Tehran, IR Iran. Tel: +98-2122180045, \\ E-mail: a.pourshahbaz@uswr.ac.ir
}

Received: April 30, 2014; Revised: June 24, 2014; Accepted: July 5, 2014

\begin{abstract}
Background: Fundamental problems with Personality Disorders (PD) diagnostic system in the previous version of DSM, led to the revision of DSM. Therefore, a multidimensional system has been proposed for diagnosis of personality disorder features in DSM-5. In the dimensional approach of DSM-5, personality disorders diagnosis is based on levels of personality functioning (Criteria A) and personality trait domains (Criteria B).

Objectives: The purpose of this study was firstly, to examine the DSM-5 levels of personality functioning in antisocial and borderline personality disorders, and second, to explore which levels of personality functioning in patients with antisocial and borderline personality disorders can better predicted severity than others.

Patients and Methods: This study had a cross sectional design. The participants consisted of 252 individuals with antisocial $(n=122)$ and borderline personality disorders $(n=130)$. They were recruited from Tehran prisoners, and clinical psychology and psychiatry centers of Razi and Taleghani Hospitals, Tehran, Iran. The sample was selected based on judgmental sampling. The SCID-II-PQ, SCID-II and DSM-5 levels of personality functioning were used to diagnose and assess personality disorders. The data were analyzed by correlation and multiple regression analysis. All statistical analyses were performed using the SPSS 16 software.

Results: Firstly, it was found that DSM-5 levels of personality functioning have a strong correlation with antisocial and borderline personality symptoms, specially intimacy and self-directedness $(\mathrm{P}<0.001)$. Secondly, the findings showed that identity, intimacy and selfdirectedness significantly predicted antisocial personality disorder severity $(\mathrm{P}<0.0001)$. The results showed that intimacy and empathy were good predictors of borderline personality disorder severity, as well $(\mathrm{P}<0.0001)$.

Conclusions: Overall, our findings showed that levels of personality functioning are a significant predictor of personality disorders severity. The results partially confirm existing studies.
\end{abstract}

Keywords: Antisocial Personality Disorder; Borderline Personality Disorder; Severity; DSM-5

\section{Background}

The fundamental problems with the Personality Disorders (PD) diagnostic system in the previous version of DSM, such as all-or-nothing diagnostic categories, considerable heterogeneity within categories, extensive overlap or comorbidity among categories, indistinct boundaries with normal personality, and incomplete coverage of personality psychopathology, led to the revision of the DSM approach $(1,2)$. Since 2000 , after the revision of DSM-IV, PD researchers largely agree that personality pathology should be represented dimensionally rather than categorically (3). Thus, many alternative dimensional models of personality have been considered (4-7), and ongoing research has been performed to delineate the conceptual and empirical structure of personality traits in the pathological range $(6,8)$. Finally, a multidimensional system was proposed for representing and diagnosis of personality disorder features in DSM-5.

The new approach for the assessment of personality pathology identifies core impairments in levels of personality functioning, pathological personality traits, and prominent personality pathology types (9). All parts needed for PD diagnosis. However, there is wide consensus that severity assessment is essential for any dimensional system of personality psychopathology (10). Moreover, the DSM-5 Personality Disorders Work Group has proposed impairments in personality functioning as the central element of PD (11). Thus, in the dimensional model of personality disorder any PD (schizotypal, antisocial, borderline, narcissistic, obsessive-compulsive and avoidant) is associated with fundamental disturbances 
of self and interpersonal relations, problems with identity, self-direction, empathy and intimacy. The review of literature showed that levels of personality functioning or self-other approach for diagnosis are informative in determining both the type and the severity of personality pathology (12-14). The severity of impairment in personality functioning has also been shown to be important in planning treatment and predicting its outcome. Despite the good advantages and empirical background of levels of personality functioning, practical studies in clinical settings with specific PDs and cross-cultural studies in this field are continuously needed $(9,12,14,15)$. Also, researchers need to determine which levels of personality functioning better predict and explain severity than others.

\section{Objectives}

The purpose of the current study was firstly, to examine the relationship between DSM- 5 levels of personality functioning on an Iranian sample with antisocial and borderline personality disorders, and second, to explore which levels of personality functioning in patients with antisocial and borderline personality disorders can better predict severity.

\section{Patients and Methods}

\subsection{Study Population and Sampling}

This study had a cross sectional study design. Of the 300 participants, 252 individuals with antisocial $(n=122)$ and borderline personality disorders $(n=130)$ were selected from September to November 2013. With regard to $\alpha=$ 0.05 and $\beta=80 \%$, four variables were tested by multiple regression analysis. Based on partial $\mathrm{R}^{2}=0.095$, the sample size for Antisocial Personality Disorder (ASPD) was calculated to be 119. For Borderline Personality Disorder (BPD), $\alpha$ and $\beta$ were 0.05 and $80 \%$, respectively, and four variables were tested by multiple regression analysis. Based on partial $\mathrm{R}^{2}=0.1$, the adequate sample size for BPD was 113. Participants were selected from prisoners (48.8\%), outpatients (16.5\%) and inpatients (7.5\%). They were recruited from Tehran prisons, and clinical psychology and psychiatry centers of Razi Psychiatric Hospital and Taleghani General Hospital, Tehran, Iran.

The sample was selected based on judgmental sampling (Expert sampling). Inclusion criteria were diagnosis of antisocial and borderline personality disorders (for personality disorders), being at least 18 years old, having at least secondary education, and the exclusion criteria were presence of a psychotic disorder, presence of severe mood disorder, presence of mental retardation, and presence of physical condition that impairs the person's mental state. Participants were 90.5\% males and 9.5\% females, aged 18 to 60 years ( $S D= \pm 1.37$ ), with guidance school degree education level or higher. Disorders of Axis
I; 106 patients (42.06\%) had no impairment, 78 patients (30.97\%) had a history of substance-related disorders, 35 patients $(13.88 \%)$ had mood disorder, 15 patients $(5.96 \%)$ had anxiety disorders, 8 patients (3.17\%) had had a history of psychotic spectrum disorder and 10 patients (3.96\%) had other disorders.

\subsection{Materials}

Data gathering measurements included psychological reports, SCID-II-PQ, SCID-II, and DSM-5 levels of personality functioning checklist.

\subsubsection{SCID}

The SCID and its versions are considered to be the most comprehensive of the structured diagnostic interviews, which are currently available. In fact, in 1987 they were new and wide range of instruments, by Spitzer, Gibbon, Williams and built in compliance with the criteria of the DSM-IV (16). This instrument has been established as the gold standard for reliable assessment of psychiatric disorders. Inter-rater reliability for SCID-I was above 70 for mood, anxiety, schizophrenic disorders and alcohol abuse; it was somewhat lower for a few other disorders (17), while for SCID-II it was reported between 0.48 and 0.98 for the categorical diagnoses (Cohen's $\kappa$ ) and 0.90 to 0.98 for the dimensional judgments (intra-class correlation coefficient) (18). Cronbach's $\alpha$ was found between 0.71 and 0.94 for the SCID-II personality disorder scales (18). Due to high accuracy of the diagnostic criteria and extraordinary compliance with DSM-IV criteria, translated to and adapted with different languages. In Iran SCID-II and SCID-II-PQ have been translated and adapted by Mohammadkhani et al. (19). The duration of the SCID-I is 30 to 90 minutes, the duration of the SCID-II is 30 to 60 minutes. Severity was determined in this study by the scores obtained from SCID-II.

\subsubsection{The DSM-5 levels of personality functioning scale}

levels of personality functioning refer to the core capacities of personality-related self and interpersonal functioning and determining the severity of any impairment in these areas (12). The DSM-5 levels of personality functioning include identity, self-direction, empathy and intimacy. If there is no impairment of these functions, personality disorders are not considered. The levels of personality functioning scale use each of these elements to differentiate five levels of impairment on a continuum of severity ranging from no impairment, i.e. healthy functioning (Level = 0), to extreme impairment (Level = 4) (11). The more impairment in these areas, the more likely that person has PD. In preliminary analysis of a sample of 424 psychiatric patients, Morey et al.(14) found that a score of greater than three (out of five) on a short five-item scale, 
had a sensitivity of $79 \%$ and a specificity of $54 \%$ for a semistructured interview diagnosis of PD.

Amini et al. (20) have translated DSM-5 levels of personality functioning scale to Farsi, and developed a semi-structured interview. Inter-rater reliability for semi-structured interview of DSM-5 levels of personality functioning items was above 0.80. The DSM-5 levels of personality functioning scale domains correlation with DSM-IV was between 0.30 and 0.69 . The duration of the semi-structured interview for DSM-5 levels of personality functioning is 15 to 25 minutes.

\subsection{Procedure}

In the implementation process, the researchers included three post-graduates of clinical psychology. To avoid probable bias outcome from these people, they were not informed of the exact goal of the research in detail and they were told that the research goal was to study personality disorders. They were entirely uninformed of the concerned disorder types. To control the probable bias, the research associates began to collect data periodically per step while they were blinded to the outcome of the previous or next step.

The colleagues were trained to use these instruments. After training under the supervision of the researcher, some people were actually interviewed, and the interviewers bug was fixed. As mentioned above, there were two groups of patients (patients with personality disorders and normal subjects). Prior to the research onset, the participants were fully informed and provided a written consent. To avoid fatigue and to increase motivation in subjects, study for each subject was conducted during two days. In the days following the completion of the demographic questionnaire, participants completed the SCID-II-PQ. Furthermore, cases, which had both symptoms of antisocial and borderline personality disorders, were examined by the Structured Clinical Interview for Personality Disorders (SCID-II). After the definitive diagnosis of antisocial personality disorder and borderline, they were invited to attend the next stage of the interview process based on DSM-5 levels of personality functioning. Ethics approval was obtained from the University of Social Welfare and Rehabilitation Sciences Research Ethics Committee, and the registered ethical code was 92/801/3110/2/A.

\subsection{Statistical Analysis}

The normality of data was tested and confirmed by the Kolmogorov-Smirnov test. We computed bivariate correlations between the DSM-5 levels of personality functioning and ASPD, and BPD symptoms. To examine which levels of personality functioning in patients with antisocial and borderline personality disorders can better predicted severity, stepwise regression analysis was used, in which SCID-II results (ASPD and BPD severity) were the dependent variables and levels of personality functioning were the predictors. The analysis was done using the SPSS 16 and NCSSPASSII.

\section{Results}

The demographic characteristics of the two groups are presented in Table 1. The two groups had no significant differences in demographic characteristics.

The mean and standard deviation scores of the levels of personality functioning in antisocial and borderline personality disorder groups are showed in Table 2.

Bivariate correlation between DSM-5 levels of personality functioning (identity, self-directedness, intimacy, empathy) and antisocial and borderline personality symptoms are presented in Table 3.

\begin{tabular}{|c|c|c|}
\hline Variable & $\begin{array}{c}\text { Antisocial } \\
\text { Personality Disorder }\end{array}$ & $\begin{array}{c}\text { Borderline } \\
\text { Personality Disorder } \\
\end{array}$ \\
\hline Age, y & $30.84 \pm 6.9$ & $33.11 \pm 8.8$ \\
\hline \multicolumn{3}{|l|}{ Gender } \\
\hline Male & $118(96.7)$ & $116(89.2)$ \\
\hline Female & $4(3.3)$ & $14(10.8)$ \\
\hline \multicolumn{3}{|l|}{ Education Levels } \\
\hline Under diploma & $62(50.8)$ & $64(9.2)$ \\
\hline Diploma & $45(36.9)$ & $36(45.4)$ \\
\hline Post diploma & $7(5.7)$ & $54(56.8)$ \\
\hline $\begin{array}{l}\text { Bachelor and } \\
\text { higher }\end{array}$ & $6(6.6)$ & $33(34.7)$ \\
\hline
\end{tabular}

Table 2. The Mean and Standard Deviation Scores of the Levels of Personality Functioning in the Two Groups a

\begin{tabular}{lcc}
\hline & ASPD & BPD \\
\hline Identity & $0.86 \pm 0.89$ & $0.36 \pm 0.76$ \\
Self-directedness & $2.18 \pm 1.57$ & $1.14 \pm 1.27$ \\
Intimacy & $2.28 \pm 1.31$ & $1.9 \pm 1.06$ \\
Empathy & $2.04 \pm 1.31$ & $0.99 \pm 1.04$ \\
\hline
\end{tabular}

a Abbreviations: ASPD, Antisocial personality disorder; and BPD, Borderline Personality Disorder.

Table 3. The Correlation Between DSM-5 Levels of Personality Functioning and Antisocial and Borderline Personality Symptoms $^{\text {a }}$

\begin{tabular}{lcc}
\hline & ASPD Symptoms & BPD Symptoms \\
\hline Identity & $0.46^{\mathrm{b}}$ & 0.08 \\
Self-directedness & $0.44^{\mathrm{b}}$ & $0.16^{\mathrm{c}}$ \\
Intimacy & $0.45^{\mathrm{b}}$ & $0.40^{\mathrm{b}}$ \\
Empathy & $0.42^{\mathrm{b}}$ & 0.003 \\
\hline a Abbreviations: ASPD, Antisocial personality & disorder; and BPD, \\
Borderline Personality Disorder. & \\
b P value $<0.01$. & \\
C P value $<0.05$. &
\end{tabular}


As seen in Table 3, positive and significant correlations were found between antisocial personality symptoms and DSM-5 levels of personality functioning. The total levels are highly correlated with ASPD symptoms (P value < 0.001).

In borderline personality disorder, except identity and empathy, other variables had significant correlations with BPD symptoms (P value $<0.001$ and $<0.05$ )

To examine the relationship between DSM-5 levels of personality functioning and ASPD and BPD severity, a stepwise regression analysis was conducted, where ASPD and BPD severity were the dependent variables and levels of personality functioning (identity, self-directedness, intimacy, empathy) were the independent variables. The results of regression analysis for levels of personality functioning and ASPD severity are presented in Table 4.

As demonstrated by Table 4, the effects of three variables were significant. These results indicate that approximately $21.7 \%, 31.4 \%$, and $33 \%$ of the variance of the ASPD severity could be accounted by identity, intimacy and self-directedness, respectively. Empathy was excluded from the table. The data showed that identity, intimacy, and self-directedness significantly predicted antisocial personality disorder severity.

The results of regression analysis for levels of personality functioning and BPD severity are displayed in Table 5.

As indicated by Table 5, the effects of both variables were significant. The results showed that intimacy and empathy were good predictors of borderline personality disorder severity. The R square of regression model was 16.5\% and $25 \%$. This means that approximately $16.5 \%$ and $25.1 \%$ of the variance of the BPD severity could be accounted for by these variables.

Table 4. Regression Analysis for Levels of Personality Functioning and Antisocial Personality Disorder Severity $(n=122)^{a}$

\begin{tabular}{lcc}
\hline Variable & \multicolumn{2}{c}{ Unstandardized Coefficients } \\
\cline { 2 - 3 } & Effect $($ mean \pm SD $)$ & t Value \\
\hline Identity & $0.62 \pm 0.06$ & 9.53 \\
Intimacy & $0.56 \pm 0.08$ & 6.82 \\
Self-directedness & $0.24 \pm 0.08$ & 2.78 \\
\hline
\end{tabular}

a Dependent Variable: Antisocial Personality Disorder severity.

Table 5. Regression Analysis for Levels of Personality Functioning and Borderline Personality Disorder Severity $(\mathrm{n}=130)^{\mathrm{a}}$

\begin{tabular}{lcc}
\hline Variable & \multicolumn{2}{c}{ Unstandardized Coefficients } \\
\cline { 2 - 3 } & Effect $($ mean \pm SD $)$ & t value \\
\hline Intimacy & $0.59 \pm 0.075$ & 7.95 \\
Empathy & $-0.49 \pm 0.081$ & -0.36 \\
\hline
\end{tabular}

a Dependent Variable: Borderline Personality Disorder severity.

\section{Discussion}

The first aim of this study was to examine the DSM-5 levels of personality functioning on an Iranian sample with antisocial and borderline personality disorders. The study findings revealed that all levels of personality functioning had significant positive correlations with ASPD. This means that DSM-5 levels of personality functioning had a good relationship with antisocial personality disorder symptoms of an Iranian sample. The present findings are consistent with the findings of Zimmermann et al. (13), and Morey et al. (14). Also, the findings indicate that except identity and intimacy, other variables (selfdirectedness and empathy) had a significant correlation with borderline personality symptoms. It seems that the study population could be one of the reasons for these findings.

Previous studies indicated that severity is the most important single predictor of concurrent and prospective dysfunction in assessing personality psychopathology $(11,21,22)$. Therefore, the second aim of this study was to explore which levels of personality functioning in patients with antisocial and borderline personality disorders can better predict severity. The stepwise regression analysis results showed that identity, intimacy, and selfdirectedness are positively and significantly correlated with antisocial personality disorder severity, indicating that those with higher scores on these variables tend to have higher symptoms of ASPD. These findings are consistent with the findings of Zimmermann et al. (13), Mullins-Sweatt et al. (23), Yang et al. (24), and Schmeck et al. (25). In patients with borderline personality disorder, the analysis showed that intimacy and empathy had a significant association with borderline personality disorder severity. It means that interpersonal level of personality functioning (intimacy and empathy) could explain the severity on patients with BPD in this study.

In addition, the results showed that the DSM- 5 levels of personality functioning are an essential part of antisocial and borderline personality disorders. Also, the results revealed that levels of personality functioning were the same in other cultures and the Iranian sample.

The present study is one of the first in which researchers examined the DSM-5 levels of personality functioning relationship with severity, and is the first study on Iranian patients with personality disorders based on the DSM-5 approach.

Overall, our findings showed that the levels of personality functioning are a significant predictor of antisocial and borderline personality disorders severity. Nevertheless, there are a number of important limitations in this work and future research is required to overcome such limitations. Firstly, the results are based on a relatively small number of cases and so caution should be taken in interpreting the data. Secondly, data was gathered by a semi-structured interview designed to assess a dimensional model of personality disorders, and future work should focus on other relevant instruments. The third 
limitation of the current study was the nature of the sample, which was drawn from antisocial and borderline personality disorders. Future research should replicate findings in larger samples with multiple personality disorders. Fourth, axis I disorders were not detected and controlled in the sample. Thus, future research should examine the role of axis I disorders in personality disorders severity. Fifth, most participants in this study were males. Therefore, other research is needed to investigate DSM- 5 levels of personality functioning and severity in females. Seventh and finally, our work focused on the assessment of DSM-5 levels of personality functioning and severity in adults. Further work is needed to determine it in other age groups.

\section{Acknowledgements}

The authors would also like to acknowledge the assistance of participants who were involved in the assessments.

\section{Funding/Support}

The authors declare that this study was not support by any financial source.

\section{References}

1. Kendler KS. An historical framework for psychiatric nosology. Psychol Med. 2009;39(12):1935-41.

2. Widiger TA, Livesley WJ, Clark LA. An integrative dimensional classification of personality disorder. Psychol Assess. 2009;21(3):243-55.

3. Widiger TA, Samuel DB. Diagnostic categories or dimensions? A question for the Diagnostic and statistical manual of mental disorders. JAbnorm Psychol. 2005;114(4):494-504.

4. Widiger TA, Simonsen E. Alternative dimensional models of personality disorder: finding a common ground. In: Widiger TA, Simonsen E, Sirovatka PJ, Regier DA, editors. Dimensional Models of Personality Disorders: Refining the Research Agenda for DSM-V. Washington, D.C: American Psychiatric Association; 2006. pp. 1-22.

5. Livesley WJ, Jackson DN, Schroeder ML. Factorial structure of traits delineating personality disorders in clinical and general population samples. J Abnorm Psychol. 1992;101(3):432-40.

6. Costa PT, McCrae RR. The Five-Factor Model of Personality and Its Relevance to Personality Disorders.J Pers Disord.1992;6(4):343-59.

7. Cloninger CR, Svrakic DM, Przybeck TR. A psychobiological model of temperament and character. Arch Gen Psychiatry. 1993;50(12):975-90.

8. Krueger RF, Eaton NR, Derringer J, Markon KE, Watson D, Skodol AE. Personality in DSM-5: Helping delineate personality dis- order content and framing the metastructure. J Pers Assess. 2011;93(4):325-31.

9. Skodol AE, Bender DS, Oldham JM, Clark LA, Morey LC, Verheul R, et al. Proposed changes in personality and personality disorder assessment and diagnosis for DSM-5 Part II: Clinical application. Personal Disord. 2011;2(1):23-40.

10. Tyrer P. The problem of severity in the classification of personality disorder.J Pers Disord. 2005;19(3):309-14.

11. American Psychological Association. Rationale for the Proposed changes to the Personality Disorders in DSM-5.USA: APA; 2012.

12. Bender DS, Morey LC, Skodol AE. Toward a model for assessing level of personality functioning in DSM-5, part I: a review of theory and methods. J Pers Assess. 2011;93(4):332-46.

13. Zimmermann J, Benecke C, Bender DS, Skodol AE, Schauenburg $\mathrm{H}$, Cierpka M, et al. Assessing DSM-5 level of personality functioning from videotaped clinical interviews: a pilot study with untrained and clinically inexperienced students. J Pers Assess. 2014;96(4):397-409.

14. Morey LC, Berghuis H, Bender DS, Verheul R, Krueger RF, Skodol AE. Toward a model for assessing level of personality functioning in DSM-5, part II: empirical articulation of a core dimension of personality pathology.J Pers Assess. 2011;93(4):347-53.

15. Hopwood CJ, Malone JC, Ansell EB, Sanislow CA, Grilo CM, McGlashan $\mathrm{TH}$, et al. Personality assessment in DSM-5: empirical support for rating severity, style, and traits. J Pers Disord. 2011;25(3):305-20.

16. Groth Marnat G. Handbook of Psychological Assessment.New York: John Wiley \& sons; 2009.

17. Skre I, Onstad S, Torgersen S, Kringlen E. High interrater reliability for the Structured Clinical Interview for DSM-III-R Axis I (SCIDI). Acta Psychiatr Scand. 1991;84(2):167-73.

18. Maffei C, Fossati A, Agostoni I, Barraco A, Bagnato M, Deborah D, et al. Interrater reliability and internal consistency of the structured clinical interview for DSM-IV axis II personality disorders (SCID-II), version 2.0. J Pers Disord. 1997;11(3):279-84.

19. Mohammadkhani P, Jokar M, Jahani-tabesh O, Tamannaei-far S. Structured Clinical Interview for DSM-IV Axis II personality disorders (Persian Version). Tehran: Danjeh; 2011.

20. Amini M, Pourshabaz A, Mohammadkhani P, Khodaie Ardakani MR. The Validity of Dimensional model of DSM-V in Antisocial and Borderline Personality Disorders. University of Social Welfare and Rehabilitation; 2014.

21. Crawford MJ, Koldobsky N, Mulder R, Tyrer P. Classifying personality disorder according to severity. J Pers Disord. 2011; 25(3):321-30.

22. First MB, Bell CB, Cuthbert B. A Research Agenda for DSM-V. In: Kupfer DJ, First MB, Regier DA editors. Personality disorders and relational disorders: a research agenda for addressing crucial gaps in DSM. 2th ed. Washington, DC: American Psychiatric Association; 2002.

23. Mullins-Sweatt SN, Edmundson M, Sauer-Zavala S, Lynam DR, Miller JD, Widiger TA. Five-factor measure of borderline personality traits. J Pers Assess. 2012;94(5):475-87.

24. Yang M, Coid J, Tyrer P. Personality pathology recorded by severity: national survey. Br JPsychiatry. 2010;197(3):193-9.

25. Schmeck K, Schluter-Muller S, Foelsch PA, Doering S. The role of identity in the DSM-5 classification of personality disorders. Child Adolesc Psychiatry Ment Health. 2013;7(1):27. 